\title{
Cortical activity reduction with stimulus repetition: a whole-head MEG analysis
}

\author{
Trevor B. Penney ${ }^{\mathrm{a}, *}$, Burkhard Maess ${ }^{\mathrm{b}}$, Niko Busch ${ }^{\mathrm{c}}$, Jan Derrfuss ${ }^{\mathrm{b}}$, Axel Mecklinger ${ }^{\mathrm{b}, \mathrm{d}}$ \\ ${ }^{a}$ Department of Psychology, Chinese University of Hong Kong, New Territories, Shatin, Hong Kong \\ ${ }^{\mathrm{b}}$ Department of Neuropsychology, Max-Planck Institute of Cognitive Neuroscience, Leipzig, Germany \\ 'Institute for Psychology, Otto von Guericke University, Magdeburg, Germany \\ ${ }^{\mathrm{d}}$ Department of Psychology, Saarland University, Saarbruecken, Germany
}

Accepted 15 October 2002

\begin{abstract}
Event related field responses to line drawings of novel visual objects were recorded in a simple target detection task using whole-head magnetoencephalography. Brain-current-source-density reconstruction indicated that, relative to initial presentations, immediately repeated nontarget stimuli elicited reduced neural activity in a region of cortex extending from the parietal lobe into the superior frontal lobe in a time window from $250 \mathrm{~ms}$ and $450 \mathrm{~ms}$. There are at least two plausible accounts of this neural activity reduction between conditions. It may reflect facilitated stimulus processing due to the existence of a representation of the repeated stimulus or it may reflect differential levels of attentional allocation to initial and repeated stimulus presentations.
\end{abstract}

(C) 2002 Elsevier Science B.V. All rights reserved.

Theme: Neural basis of behavior

Topic: Cognition

Keywords: Magnetoencephalography; Event related field; Visual stimuli; Repetition effect; Memory; Attention

\section{Introduction}

Repeated exposure to a stimulus often elicits a different brain response from that elicited by the first presentation. The exact nature of the neural response, however, depends on both the task requirements and the meaning that a particular stimulus has within a given experimental context. For example, so-called suppression neurons in macaque inferotemporal cortex reduce firing when a stimulus is repeated independent of whether the repetition is task relevant or irrelevant, whereas enhancement neurons increase firing to repeated stimuli only when the stimulus repetition is task relevant [16]. Several authors have suggested that the physiological underpinning of visual perceptual priming, where perceptual priming is defined as the facilitated processing of previously seen 019 .

*Corresponding author. Tel.: +852-26-096-456; fax: +852-26-035-

E-mail address: tpenney@psy.cuhk.edu.hk (T.B. Penney). stimuli, is reflected in the activity of such cortical suppression neurons [5,28]. In brief, this view claims that a stimulus representation becomes more efficient when the stimulus is repeated because neurons that responded on initial exposure, but that are not critical for the stimulus representation, do not respond on subsequent exposures. This sharpening of the stimulus representation permits faster processing of the stimulus on subsequent presentations.

Recently, we reported an event related potential (ERP) study [18] that revealed two distinct brain responses to task-irrelevant repetition of novel visual objects. Relative to initial presentations, stimulus repetitions elicited a reduction in a negative going ERP waveform over frontal recording sites between 250 and $350 \mathrm{~ms}$. This effect was interpreted as reflecting facilitated access to neural codes, such as an episodic representation of the immediately preceding stimulus classification and/or an image-based representation of the stimulus. Stimulus repetition also elicited a reduction in a positive going waveform over 
parietal recording sites between 300 and $600 \mathrm{~ms}$. This effect was unusual because the typical parietal ERP repetition effect is one of increased amplitude in a positive going waveform (e.g. [2,19,27], see [20] for review), although there are exceptions to this pattern (e.g. $[21,26,27])$. We suggested that neurons similar in function to the suppression neurons described above mediated the parietal effect and that, psychologically, it reflected the availability of a newly formed representation in memory. This explanation assumes, however, that the attenuation of a positive parietal ERP waveform with stimulus repetition is due to an underlying reduction in neural activity. This assumption may be incorrect given that it is possible for neural sources to be oriented in such a way that increases in neural activity are manifested as reductions in positivity at the scalp [17]. Furthermore, whenever possible the location of the neural tissue responsible for a scalp recorded ERP effect should be taken into account when attributing a psychological interpretation to a neural activity change [13]. Although the source of our parietally recorded ERP effect is unknown, its broad scalp distribution suggests a deep source. Given the strong evidence implicating the inferior temporal cortex as the neural substrate for object representations (e.g. [25]), it is plausible that it originated in regions of occipital and/or temporal cortex. Moreover, Jiang et al. [11] recently demonstrated reduced brain activity, as measured by fMRI, in extrastriate visual cortex to repeated nontarget faces during a working memory task. Interestingly, Henson et al. [10], using fMRI, showed a dissociation of brain activity in a right fusiform region to repeated stimuli depending on whether the task-irrelevant stimulus repetitions were of familiar or unfamiliar stimuli. Repetitions of unfamiliar stimuli elicited enhanced responses whereas repetitions of familiar stimuli elicited attenuated responses. They proposed that repeated exposures to unfamiliar stimuli engaged additional psychological processes such as formation of new memory representations. Given that the task irrelevant nontarget stimuli used in our previous ERP study were line drawings of unfamiliar geometric objects, it is possible that one or both of the ERP differences obtained in that study reflected an increase in neural activity with repetition rather than a decrease.

In an effort to localize the neural source(s) of our ERP repetition effects, and to determine whether neural activity was reduced or increased with task irrelevant stimulus repetitions, we examined the brain response in the same task using whole head magnetoencephalography (MEG). MEG has identical temporal resolution to EEG, but has the potential to provide better spatial resolution. In addition, application of the brain surface current density (BSCD) reconstruction technique [12] to MEG data provides a measure of the amount of cortical activity for each condition, thereby allowing determination of whether brain activation increases or decreases with stimulus repetition.

\section{Materials and methods}

\subsection{Subjects}

Nineteen volunteers from the University of Leipzig (fifteen female) between 18 and 26 years of age (mean 22) participated in return for a cash payment. All participants were right-handed and had normal or corrected to normal vision.

\subsection{Apparatus}

Participants sat in a magnetically shielded dimly lit chamber and stimuli were projected (Epson EMP5000), via three mirrors, onto a 15 -inch display screen at a viewing distance of approximately $100 \mathrm{~cm}$ from the participant. Stimulus presentation was controlled by a personal computer. Participants responded to the target stimuli on a custom-built three-button response pad.

\subsection{Stimuli}

Target stimuli consisted of 34 everyday objects [24] (e.g. hammer, sailboat) and 34 scrambled figures, drawn by combining selected features from other, unpresented, everyday objects. Nontarget stimuli consisted of 224 line drawings of novel geometric objects [18].

\subsection{Procedure}

Participants were tested individually in a target detection task. Each participant performed in two separate sessions, at least 1 week apart. There were 68 target trials, and 336 nontarget trials per session. The nontarget trials consisted of 112 figures that were presented once, and 112 figures that were presented twice, with the second presentation immediately following the first. The nonrepeated stimuli from the first session were used as repeated stimuli in the second session and the repeated stimuli from the first session served as nonrepeated stimuli in the second session. The duration of stimulus presentation was $500 \mathrm{~ms}$ (1200 ms offset to onset delay). Prior to the start of the experiment, participants were shown examples of target and nontarget stimuli, and were told to simultaneously press the right and left response buttons with their right and left thumbs in response to targets and to withhold responding for nontargets.

\subsection{MEG recording and analysis}

A 148-channel whole head MEG (Magnes WHS 2500, 4-D Neuroimaging), 11 additional MEG reference channels, and four electrodes of EOG were recorded continu- 
ously with online bandpass filters $(0.1-50 \mathrm{~Hz})$ at a rate of $254.31 \mathrm{~Hz}$. Before and after each of the two sessions, the position of the sensor array with respect to the nasion-ear coordinate system was measured. All measurements were converted to the subject's mean sensor position and the recordings for each individual subject were averaged over the two sessions. A number of current sources, modeling the cortical activity of individual subjects in each condition, were reconstructed using the BSCD technique, described in detail by Knoesche et al. [12]. This technique transforms the outer magnetic field distribution to a cortical current distribution, thereby providing a measure of the amount of cortical activity for each condition. Although currents are orientation dependent, this measure depends on the magnitude of currents only. To avoid an unacceptably large number of statistical comparisons, the BSCD values were pooled to form seven regions of interest (ROIs) in each hemisphere (superior-frontal, inferior-frontal, parietal, posterior-parietal, occipital, anterior-temporal, and posterior-temporal; see Fig. 2). Four time windows, covering the range of the ERP effects obtained in an earlier study, were selected: 150-250, 250-350, 350-450 and 450-550 ms. ANOVA was used and all effects with two or more degrees of freedom in the numerator were adjusted for violations of sphericity according to the Greenhouse-Geisser formula [8].

\section{Results}

\subsection{Behavioral data}

On average, the subjects correctly identified more than $99 \%$ of the targets and made false alarms to less than $2 \%$ of the nontargets. The average response time to targets was $451 \mathrm{~ms}$.

\subsection{MEG BCSD analysis}

Time course plots of the BSCD in each of the left and right hemisphere regions of interest for the first and second presentations of nontarget stimuli are presented in Fig. 1. An initial four-way ANOVA on these BSCD values, treating Time Window, Hemisphere, ROI, and Presentation as repeated measures factors, revealed a significant main effect of Presentation, $F(1,18)=5.98, P<0.05$, and significant Time Window $\times$ Presentation, $F(3,54)=3.09, P<0.05$, ROI $\times$ Presentation, $F(6,108)=4.94, P<0.05$, and Time

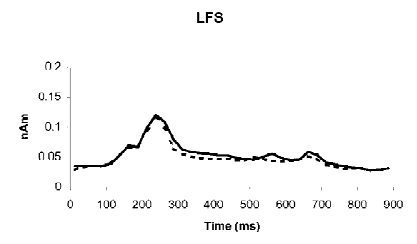

LFI

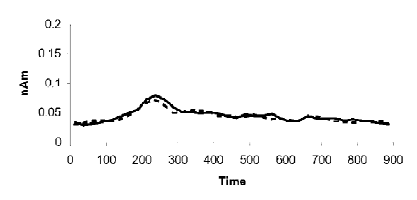

LPA

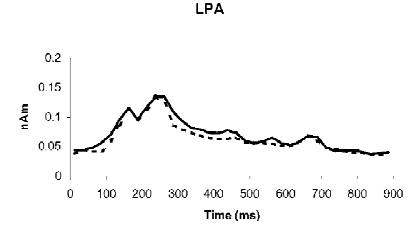

LTP

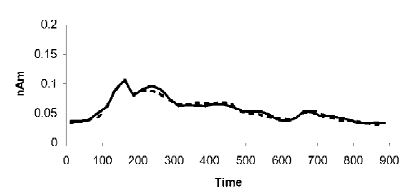

RFS

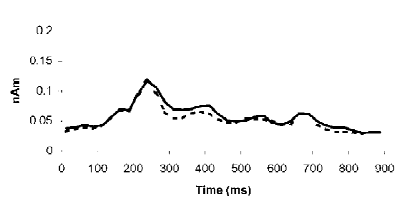

RFI

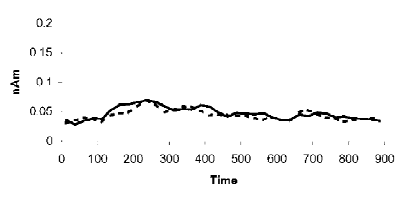

RPA

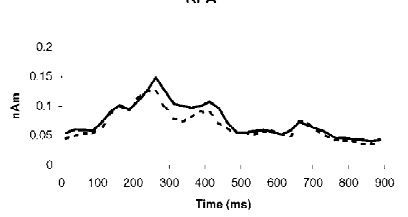

RTP

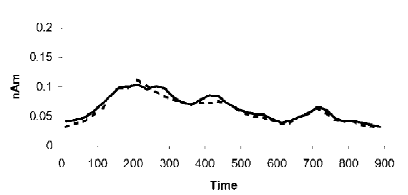

LoS

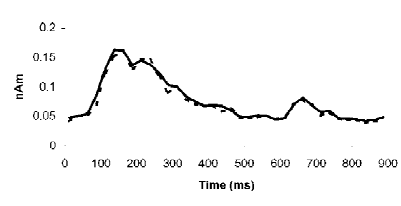

Lol

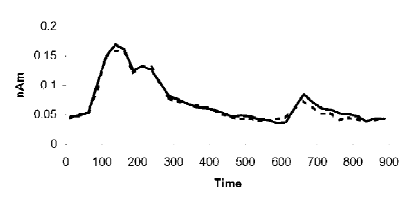

LTA

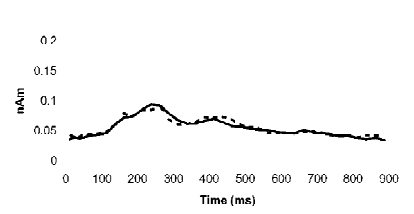

ROS

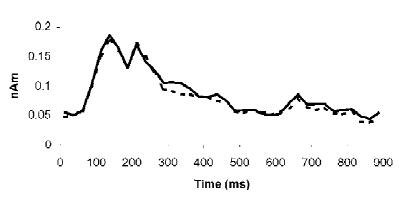

ROI

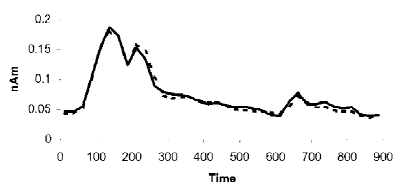

RTA

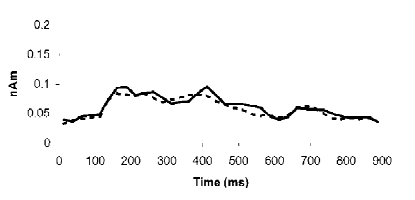

Fig. 1. Plots of the BSCD, in nAm, for the first (solid line) and second (dashed line) presentations of nontarget stimuli, in a time window from stimulus onset (0 ms) to $900 \mathrm{~ms}$ thereafter. BCSD is plotted for each of the 14 regions of interest in the present study. L, left hemisphere; R, right hemisphere; FS, frontal superior; FI, frontal inferior; PA, parietal; TP, posterior temporal; OS, occipital superior; OI, occipital inferior; TA, temporal anterior. 
Window $\times$ ROI $\times$ Presentation, $F(18,324)=3.25, P<.05$, interactions. Follow-up two-way ANOVAs, treating Presentation and ROI as repeated measures factors for each time window separately, and collapsing over hemisphere, revealed that the effects of Presentation, and the Presentation $\times$ ROI interactions were nonsignificant in the 150-250-ms and the 450-550-ms time windows. From 250 to $350 \mathrm{~ms}$, and 350 to $450 \mathrm{~ms}$, however, the Presentation $\times$ ROI interactions were significant, $F(6$, 108) $=6.08$ and 5.93, respectively, both $P<0.01$. Subsequent one-way repeated measures ANOVAs were conducted for each ROI, collapsed across hemisphere, for the 250-350-ms and 350-450-ms time windows. From 250 to $350 \mathrm{~ms}$, second presentations resulted in reduced BSCD over the frontal superior, posterior-parietal, and the parietal ROIs, $F(1,18)=20.49,17.94$, and 43.69, respectively, all $P \leq 0.01$. From 350 to $450 \mathrm{~ms}$, second presentations elicited reduced BSCD over the frontal superior and the parietal ROIs, $F(1,18)=12.98$, and 22.42 , respectively, all $P<0.01$. Inspection of Fig. 2 suggests that these neural activity reductions had a parietal accentuation and extended into neighboring regions of posterior parietal and superior frontal cortex, rather than comprising independent effects.

\section{Discussion}

The main result of the present study is that, compared to initial presentations, task-irrelevant repetitions of nontarget novel visual stimuli elicited a neural activity reduction between 250 and $350 \mathrm{~ms}$ in the posterior-parietal, parietal, and superior-frontal ROIs and between 350 and $450 \mathrm{~ms}$ in the parietal and the superior frontal ROIs. One goal of the present study was to clarify the results obtained in an earlier ERP study. The correspondence of effects between the MEG and ERP studies is supported by the similar onset latency of effects in the two studies. Moreover, the spatial distribution of the repetition related activity reduction in the MEG experiment is similar to the distribution of electrode locations that showed repetition related differences in the ERP study. In the ERP study, however, distinct effects were obtained over frontal and parietal electrode sites. At frontal sites there was a decrease in a negative going waveform whereas at parietal sites there was a decrease in a positive going waveform. In the present experiment, there was a widespread cortical activity reduction that failed to statistically dissociate into distinct frontal and parietal cortex components. It is possible that the difference between studies is due to the
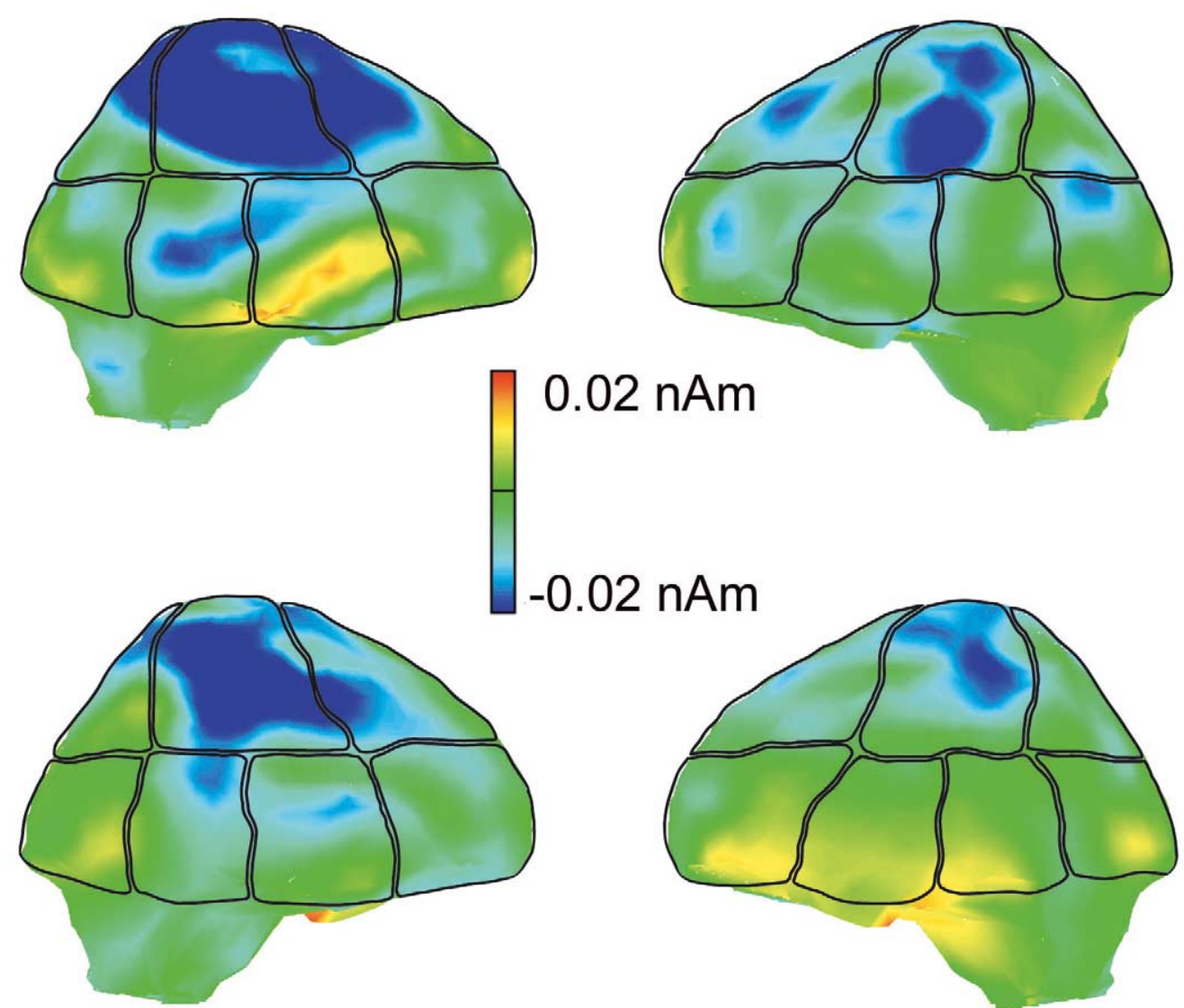

$-0.02 \mathrm{nAm}$

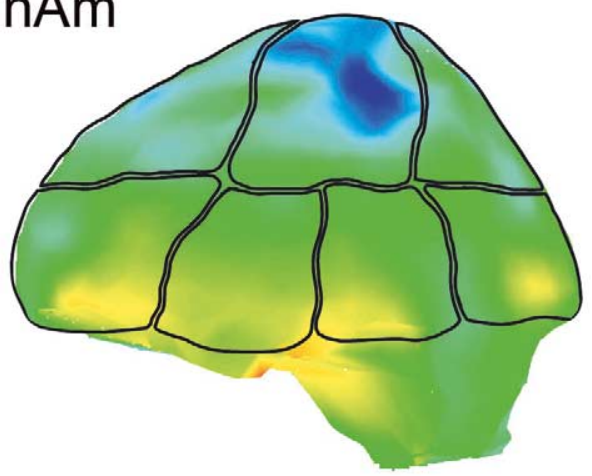

Fig. 2. Grand average BSCD difference maps (nontarget presentation 2 nontarget presentation 1 ), in nAm, in time windows from 250 to 350 ms (top row) and 350-450 ms (bottom row). Blue coloration indicates reduced brain activity with stimulus repetition. Approximate areas of the regions of interest (ROIs) used in the statistical analyses are indicated by black outlines. 
differential sensitivity EEG and MEG measures have to tangential and radial neural sources. In any case, the results suggest that both the frontal and the parietal differences in the earlier ERP study were due to reductions in neural activity.

As mentioned in the Introduction, researchers using single cell recording in macaque monkeys have associated task irrelevant stimulus repetitions with decreases in neural activity, whereas active recognition processes have been associated with increases in neural activity [16]. In addition, a large number of PET and fMRI studies of recognition have associated active recollection with increases in blood-flow dependent measures of brain activity (see [22] for review). Therefore, the present fronto-parietal neural activity reduction is consistent with facilitated stimulus processing of the repeated stimuli (cf. [28]) rather than active recollection of the stimuli, or the creation of new memory representations for unfamiliar stimuli (cf. [10]). We had expected to localize activity reduction over the temporal cortex because of the strong evidence implicating the inferior temporal cortex as the neural substrate for object representations (e.g. [25]). However, caution must be applied in interpreting this null result because the larger distance between MEG squids and the ventral temporal lobe renders the MEG BCSD technique relatively insensitive to these locations.

It is not obvious what cognitive process a parietally focused cortical activity reduction that occurs in response to task-irrelevant stimulus repetitions might reflect. The standard views of parietal cortex function include the ideas that it is primarily involved in spatial processing [25] and intermodal integration processes [1], but there is also recent evidence of shape selective neurons in the primate parietal cortex [23] and neuropsychological studies have demonstrated object shape sensitivity in the human parietal cortex (see [7] for review). Consequently, it is plausible that the activity reduction obtained here reflects modulation of stimulus representations. As noted in the Introduction, a stimulus representation may become more efficient with stimulus repetition because neurons that respond on initial stimulus exposure, but that are not critical for the stimulus representation, may not respond on subsequent exposures.

Although a parietally localized memory representation may be plausible, it is necessary to consider alternative accounts for the repetition related activity reduction. Neuronal activity in the parietal cortex, particularly in the right hemisphere, is modulated by allocation of spatial attention ([6] see [3] for review). Therefore, one might argue that the neural activity reduction obtained here occurred because on repetition trials participants allocated less attention to the computer screen location where stimuli were presented. However, an intentional reduction of the amount of attention allocated on repeated trials prior to stimulus presentation requires participants to be able to predict whether a nontarget stimulus would be repeated or not. This seems unlikely given that $50 \%$ of the nontarget stimuli were immediately repeated and $50 \%$ were not, meaning that most of the time a current nontarget stimulus was not a valid predictor of the stimulus category of the subsequent stimulus. Of course, repeated stimuli were always followed by an initial presentation of another stimulus so these stimuli did have some predictive validity.

A related possibility is that allocation of attention to the stimulus location or to the stimulus itself was modulated after stimulus onset. Shifts of spatial attention have been associated with increased blood-flow to regions of frontal and parietal cortex $[4,9]$, and nonspatial attention shifts have been associated with activation of parietal cortex [15]. If presentation of a novel stimulus elicited increased attention as compared to a repeated stimulus, then one would expect differential parietal cortex activity between the two conditions.

In order for attention to be differentially allocated based on stimulus repetition, the stimuli must be represented and the brain system responsible for attentional allocation must be sensitive to those representations and whether they change across trials. Interestingly, working memory and spatial attention tasks have been shown to activate common fronto-parietal cortical regions and this shared network has been attributed the function of shifting attentional focus across space, time, or cognitive domains [14].

The present results are theoretically interesting because they show that neural activity in parietal and superior frontal cortex is reduced in response to task-irrelevant stimulus repetition. In doing so, they provide support for an earlier claim that the ERP repetition effect obtained with the same task reflects reduced brain activation, and also show that the parietal cortex is critical for generating this effect. Localization of an activity reduction to this area was unexpected however, and as such raises questions about the cognitive process or processes underlying the effect. As described above, one possibility is that the activity reduction reflects facilitated processing of the repeated stimuli. An alternative possibility is that repeated stimuli attract less attention as compared to first presentations and that the fronto-parietal activity reduction to repeated nontargets is a reflection of reduced attention. The present data, however, do not allow us to legislate between these two possibilities.

\section{Acknowledgements}

We thank Ulrich Oertel and Yvonne Wolff for their assistance and Annett Schirmer for comments on an earlier version of this article.

\section{References}

[1] R.A. Andersen, A.P. Batista, L.H. Snyder, C.A. Buneo, Y.E. Cohen, Programming to look and reach in the posterior parietal cortex, in: 
M.S. Gazzaniga (Ed.), The New Cognitive Neurosciences, MIT Press, Cambridge, MA, 2000, pp. 515-524.

[2] M. Besson, M. Kutas, C. Van Petten, An event-related potential (ERP) analysis of semantic congruity and repetition effects in sentences, J. Cognit. Neurosci. 4 (1992) 132-149.

[3] C.L. Colby, M.E. Goldberg, Space and attention in the parietal cortex, Annu. Rev. Neurosci. 22 (1999) 319-349.

[4] M. Corbetta, J.M. Kincade, J.M. Ollinger, M.P. McAvoy, G.L. Shulman, Voluntary orienting is dissociated from target detection in human posterior parietal cortex, Nature Neurosci. 3 (2000) 292 297.

[5] R. Desimone, Neural mechanisms for visual memory and their role in attention, Proc. Natl. Acad. Sci. USA 93 (1996) 13494-13499.

[6] D.R. Gitelman, A.C. Nobre, T.B. Parrish, K.S. LaBar, Y.H. Kim, J.R. Meyer, M.M. Mesulam, A large-scale distributed delineation network for covert spatial attention Further anatomical delineation based on stringent behavioral and cognitive controls, Brain 122 (1999) 1093-1106

[7] M.A. Goodale, Perception and action in the human visual system, in: M.S. Gazzaniga (Ed.), The New Cognitive Neurosciences, The MIT Press, Cambridge, MA, 2000, pp. 365-377.

[8] S. Greenhouse, S. Geisser, On methods in the analysis of profile data, Psychometrika 24 (1959) 95-112.

[9] J.B. Hopfinger, M.H. Buonocore, G.R. Mangun, The neural mechanisms of top-down attentional control, Nature Neurosci. 3 (2000) 284-291.

[10] R. Henson, T. Shallice, R. Dolan, Neuroimaging evidence for dissociable forms of repetition priming, Science 287 (2000) 12691272.

[11] Y. Jiang, J.V. Haxby, A. Martin, L.G. Ungerleider, R. Parasuraman, Complementary neural mechanisms for tracking items in human working memory, Science 287 (2000) 643-646.

[12] T.R. Knosche, B. Maess, A.D. Friederici, Processing of syntactic information monitored by brain surface current density mapping based on MEG, Brain Topogr. 12 (1999) 75-87.

[13] M. Kutas, A. Dale, Electric and magnetic readings of mental functions, in: M.D. Rugg (Ed.), Cognitive Neuroscience, The MIT Press, Cambridge, MA, 1997, pp. 197-242.

[14] K.S. LaBar, D.E. Gitelman, T.B. Parrish, M.M. Mesulam, Neuroanatomic overlap of working memory and spatial attention net- works: a functional MRI comparison within subjects, NeuroImage 10 (1999) 695-704.

[15] T.H. Le, J.V. Pardo, X. Hu, 4 T-fMRI study of the nonspatial shifting of selective attention: cerebellar and parietal contributions, J. Neurophysiol. 79 (1998) 1535-1548.

[16] E.K. Miller, R. Desimone, Parallel neuronal mechanisms for shortterm memory, Science 263 (1994) 520-522.

[17] P.L. Nunez (Ed.), Neocortical Dynamics and Human EEG Rhythms, Oxford University Press, New York, 1995.

[18] T.B. Penney, A. Mecklinger, D. Nessler, Repetition related ERP effects in a visual object target detection task, Cognit. Brain Res. 10 (2001) 239-250

[19] M.D. Rugg, Event-related brain potentials dissociate repetition effects of high- and low-frequency words, Mem. Cognit. 18 (1990) 367-379.

[20] M.D. Rugg, ERP studies of memory, in: M.D. Rugg, M.G.H. Coles (Eds.), Electrophysiology of Mind, Oxford University Press, Oxford, 1995, pp. 132-170.

[21] M.D. Rugg, M. Soardi, M.C. Doyle, Modulation of event-related potentials by the repetition of drawings of novel objects, Cogn. Brain Res. 3 (1995) 17-24.

[22] D.L. Schacter, A.D. Wagner, Medial temporal lobe activations in fMRI and PET studies of episodic encoding and retrieval, Hippocampus 9 (1999) 7-24.

[23] A.B. Sereno, J.H.R. Maunsell, Shape selectivity in primate lateral intraparietal cortex, Nature 395 (1998) 500-503.

[24] J.G. Snodgrass, J.M. Vanderwart, A standardized set of 260 pictures: norms for name agreement, image agreement, familiarity, and visual complexity, J. Exp. Psychol.: Human Learn. Mem. 6 (1980) 174 215.

[25] L.G. Ungerleider, J.V. Haxby, 'What' and 'where' in the human brain, Curr. Opin. Neurobiol. 4 (1994) 157-165.

[26] C. Van Petten, M. Kutas, R. Kluender, M. Mitchener, H. McIsaac, Fractionating the word repetition effect with event-related potentials, J. Cognit. Neurosci. 3 (1991) 131-150.

[27] C. Van Petten, A.J. Senkfor, Memory for words and novel visual patterns: Repetition, recognition, and encoding effects in the eventrelated brain potential, Psychophysiology 33 (1996) 491-506.

[28] C.L. Wiggs, A. Martin, Properties and mechanisms of perceptual priming, Curr. Opin. Neurobiol. 8 (1998) 227-233. 